\title{
Hollow Electron Ptychographic Diffractive Imaging
}

\author{
Biying Song, ${ }^{1}$ Zhiyuan Ding, ${ }^{1}$ Christopher S. Allen, ${ }^{2,3}$ Hidetaka Sawada, ${ }^{4}$ Fucai Zhang, ${ }^{5}$ Xiaoqing Pan, ${ }^{6}$ \\ Jamie Warner, ${ }^{2}$ Angus I. Kirkland, ${ }^{2,3}$ and Peng Wang ${ }^{1, *}$ \\ ${ }^{1}$ National Laboratory of Solid State Microstructures, College of Engineering and Applied Sciences \\ and Collaborative Innovation Center of Advanced Microstructures, Nanjing University, Nanjing 210093, People's Republic of China \\ ${ }^{2}$ Department of Materials, University of Oxford, Parks Road, Oxford OXI 3PH, United Kingdom \\ ${ }^{3}$ Electron Physical Sciences Imaging Centre, Diamond Lightsource Ltd., Didcot, Oxfordshire OX11 ODE, United Kingdom \\ ${ }^{4} J E O L$ Ltd, 1-2 Musashino, 3-Chome, Akishima, Tokyo 196, Japan \\ ${ }^{5}$ Department of Electrical and Electronic Engineering, Southern University of Science and Technology, \\ Shenzhen 518055, People's Republic of China \\ ${ }^{6}$ Department of Chemical Engineering and Materials Science and Department of Physics and Astronomy, University of California, \\ Irvine, California 92697, USA
}

(Received 28 May 2018; published 1 October 2018)

\begin{abstract}
We report a method for quantitative phase recovery and simultaneous electron energy loss spectroscopy analysis using ptychographic reconstruction of a data set of "hollow" diffraction patterns. This has the potential for recovering both structural and chemical information at atomic resolution with a new generation of detectors.
\end{abstract}

DOI: $10.1103 /$ PhysRevLett.121.146101

Introduction.-Scanning transmission electron microscopy (STEM) has been extensively used for structural analysis in physical, chemical, and biological sciences for several decades [1]. High-angle annular dark field (HAADF) STEM images show a strong atomic number $(Z)$, contrast and a significant advantage of STEM-HAADF imaging is that it allows simultaneous electron energy loss spectroscopy (EELS) due to the annular detector geometry used, thus providing composition and structural information at atomic resolution [2-4]. However, HAADF images are relatively insensitive to light elements in the presence of heavier elements [1,5]. More recently, it has been demonstrated that differential phase contrast (DPC) STEM imaging using solid state segmented detectors can be related to the atomic-scale electric field [6]. However, a method compatible with quantitative phase recovery and simultaneous EELS analysis has not been demonstrated yet.

Ptychography was originally proposed by Hoppe [7] using arrays of far-field diffraction patterns formed by a known, finite-sized probe scanned over the sample with a partial overlap of the probe positions. This overlap of illuminated regions provided a redundancy, enabling a convergent iterative reconstruction of the exit wave [8,9], thereby resulting in a unique phase solution. The greatest potential advantage of this technique is that the resolution of reconstructions is not limited by aberrations of the optical system, but is instead determined by the maximum spatial frequency recorded in diffraction patters and the stability and accuracy of scan positions. Ptychographic coherent diffractive imaging has been successfully implemented using both visible light $[10,11]$ and $\mathrm{x}$-ray sources
[12-14]. Using electrons, the experimental geometry required is similar to that for aberration-corrected STEM imaging and high-atomic resolution phase-sensitive information has been successfully reconstructed at both low and intermediate voltages [15-20]. Importantly, Brown et al. have demonstrated that only a limited portion of electrons detected are required for reconstruction [18]. Wang et al. [19] have also reported a ptychographic reconstruction of a $\mathrm{LaB}_{6}$ crystal in which light $B$ atoms were clearly resolved together with heavy La atoms in reconstructed phases. An alternative technique involves scanning a focused probe in a highly convergent beam geometry and extracting phase information by evaluating the overlap between the first- and zero-order scattered intensity using direct methods [21-27] rather than an iterative method [10]. Using these direct methods, Nellist et al. have reconstructed phase information at atomic resolution [22-24,26]. Importantly, in this geometry the focused probe used is compatible with the conditions required for simultaneous incoherent $Z$-contrast imaging.

Recent advances in fast direct electron detectors and dataprocessing algorithms have triggered a renewed interest in phase-retrieval techniques [18,24,26-29]. In this Letter, using a fast direct counting detector [30], we propose a new configuration for iterative ptychographic reconstruction from hollow diffraction patterns using a focused scanned probe. Importantly, this will enable the future combination of ptychography with both EELS and HAADF to simultaneously provide high-efficiency phase and $Z$ contrast imaging together with spectroscopic information in a single experiment. We demonstrate the feasibility of this method 


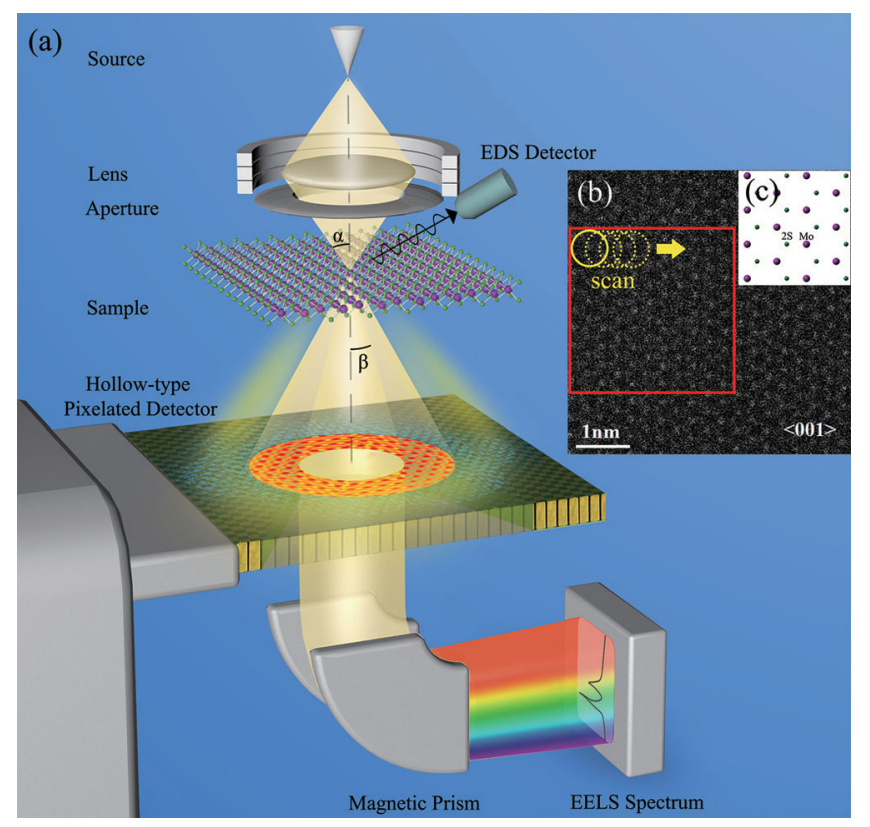

FIG. 1. Schematic illustration of the experimental setup used. (a) Optical configuration used for ptychographic data acquisition. ( $\alpha$ is the probe convergence semiangle and $\beta$ is the semiangle of the hollow region of detector.) (b) HAADF image of $\mathrm{MoS}_{2}$ oriented along the [001] direction. The region enclosed in the red box was subsequently reconstructed using the ePIE algorithm. (c) Projected atomic model of $\mathrm{MoS}_{2}$ displayed along the [001] direction.

by retrieving phases from synthesized hollow diffraction patterns experimentally acquired from a monolayer $\mathrm{MoS}_{2}$ sample and have compared these with STEM-ADF imaging. Furthermore, we have performed multislice simulations to evaluate the accuracy of these experimental ptychographic results and explored how the effects of the experimental scan step size and the probe position error influence hollow ptychographic reconstructions.

Experimental data acquisition.-The microscope used in these studies was a JEOL ARM 300CF located at the Electron Physical Sciences Imaging Centre and operated at $80 \mathrm{kV}$ with a convergence semiangle, $\alpha=24 \mathrm{mrad}$. The ptychographic data sets reported here were obtained from a monolayer $\mathrm{MoS}_{2}$ located at the probe focus plane [Fig. 1(a)]. Further details of the experimental configuration are provided in the Supplemental Material, part I [31]. As an alternative to using the entire bright-field disk of each diffraction pattern for ptychographic reconstruction [19], we propose collecting a hollow cone diffraction pattern from a future physical or synthetic hollow pixelated detector (hollow ptychography), the central hollow part of which would allow electrons to enter an EELS spectrometer. However, the required hollow detector hardware is not currently available and hence as a proof of concept, we have collected full diffraction patterns using a MEDIPIX3 detector [30] and mimicked hollow acquisition by applying a mathematically synthesized hollow mask for different

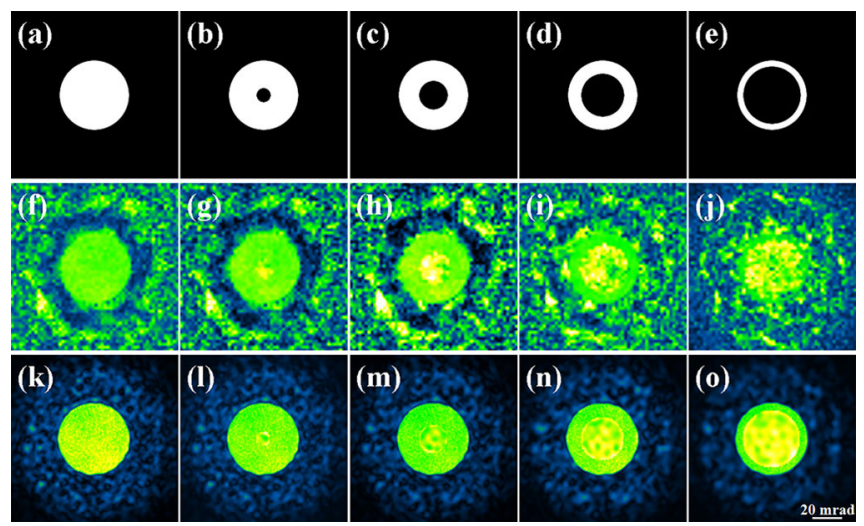

FIG. 2. (a)-(e) Binary masks applied to diffraction patterns with hollow inner angles of (a) 0, (b) 5, (c) 10, (d) 15, and (e) $20 \mathrm{mrad}$ and an outer angle of $24 \mathrm{mrad}$, which is equal to the convergence semiangle $\alpha$. Diffraction patterns calculated from the object function reconstructed from (f)-(j) experimental and (k)-(o) simulated data set with different hollow inner angles corresponding to the masks shown in (a)-(e), respectively. All diffraction patterns were obtained after 50 reconstruction iterations. The 20-mrad scale bar applies to all data in (a)-(o).

inner angles $\beta$, as shown in Figs. 2(a)-2(e), before reconstruction using the extended ptychographic iterative engine (ePIE) algorithm. The effective area of diffraction patterns used corresponded to the bright annular region of masks, as shown in Figs. 2(a)-2(e). Further details of the calculation of the masks used are provided in the Supplemental Material, part II [31].

The initially generated estimate of the focused probe is shown in Fig. S3(a). Further details are given in the Supplemental Material, part III [31]. For the experimental step size used, the overlap ratio between adjacent probe positions was approximately $89 \%$ [8]. Because of this large overlap ratio and the number of diffraction patterns recorded, the missing information in the center of each diffraction pattern could be retrieved by performing multiple iterations of the ePIE algorithm. This retrieval can be observed by examining the evolution of calculated diffraction patterns of the object function reconstructed from both experimental [Figs. 2(f)-2(j)] and simulated [Figs. 2(k)-2(o)] data set after 50 iterations. Using the ePIE algorithm [10,39], the exit wave was assumed to be a product of probe and object transmission functions, which is satisfied for a relatively weakly scattering sample such as monolayer $\mathrm{MoS}_{2}$.

Comparison of conventional system imaging and ptychography.-Figure 3(a) shows an annular dark field (ADF) image, synthesized directly from the full ptychographic data sets by integrating the intensity over angles from 24 to $32 \mathrm{mrad}$ (the maximum acquisition angle of the detector). Note that the dose used to record this image was identical to that used for ptychographic reconstructions. Using the ePIE algorithm, both the conventional (Con Ptycho, using the full diffraction pattern) [Fig. 3(b)] and 


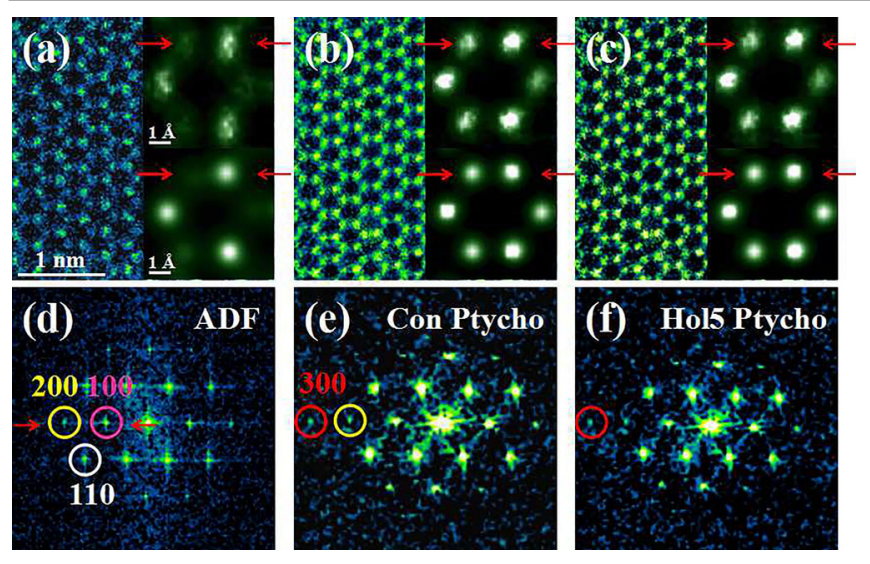

FIG. 3. (a) ADF image of monolayer $\mathrm{MoS}_{2}$. (b) Conventional ptychographic (Con Ptycho, full diffraction pattern) and (c) Hollow ptychographic (Hol5 Ptycho, $\beta=5 \mathrm{mrad}$ ) reconstructed phases from the same sample in the region enclosed by the red square in Fig. 1(b), respectively. The top-right and bottomright insets in (a)-(c) are the corresponding experimental unit-cell averaged and simulated results, respectively. The $1-\mathrm{nm}$ and $1-\AA$ scale bars apply to (a)-(c) and their corresponding insets, respectively. (d)-(f) Power spectra of (a)-(c). The pink, white, yellow, and red circles mark (100), (110), (200), and (300) reflections corresponding to spacings of $273,158,136$, and $91 \mathrm{pm}$, respectively.

hollow ptychographic reconstruction (Hol 5 Ptycho, with a hollow mask of a 5-mrad inner angle) [Fig. 3(c)] were calculated. The $2 S$ (double $S$ ) atoms were better resolved in both reconstructed ptychographic phases than in the ADF image at a dose of $7.5 \times 10^{4} e / \AA^{2}$, suggesting that ptychographic reconstructions are more robust under low-dose conditions. The top-right insets in Figs. 3(a)-3(c) show the corresponding unit-cell averages of these results. Figures 3(d)-3(f) show the associated power spectra calculated from Figs. 3(a)-3(c) with the (100), (110), and (200) reflections corresponding to spacings of 273 , 158 , and $136 \mathrm{pm}$ clearly present. The power spectra calculated from the reconstructed complex object waves [Figs. 3(e)-3(f)] show an additional weak (300) reflection corresponding to a spacing of $91 \mathrm{pm}$, and the lower order reflections are also stronger than those in the power spectra calculated from the ADF image. These results demonstrate that the resolution in the complex object wave functions reconstructed from both conventional and hollow ptychography is $0.91 \AA$. This resolution can be understood by considering diffraction patterns calculated from the reconstructed object functions [Figs. 2(f)-2(j)], which show that high-angle scattering is recorded in the dark field region at up to two times the convergence semiangle $\alpha$.

Furthermore, we have compared ADF and ptychographic results in real and reciprocal space. Figure S5(a) shows line profiles across a pair of Mo and $2 S$ atom positions. Compared with ptychographic phases, the signal at Mo and $2 S$ positions in an ADF image is close to the noise level, consistent with the lower contrast in an ADF image. Figure S5(b) shows the corresponding intensity profiles from the position indicated by red arrows in the power spectra, clearly demonstrating that the ptychographic phases show an additional (300) reflection corresponding to a spacing of $91 \mathrm{pm}$.

Comparison of experimental and simulated results.-To confirm our interpretation of the above experimental results, we have performed a series of theoretical ptychographic reconstructions using synthetic hollow diffraction patterns simulated using the multislice method [38] from a monolayer $\mathrm{MoS}_{2}$ model. Further details of the simulation parameters are provided in the Supplemental Material, part IV [31]. A simulated ADF image together with conventional and hollow ptychographic phases $(\beta=5 \mathrm{mrad})$ are shown in the bottom-right insets in Fig. 3 from left to right. Line profiles obtained across a pair of Mo and $2 S$ atom positions are shown in Fig. S5(c), which demonstrates that the contrast difference between $2 S$ and Mo positions in ptychographic reconstructions is higher than in the ADF image. The complete set of simulated reconstructed phases with various hollow angles are shown in Fig. S6.

Figures S7(a) and S7(b) show line profiles across Mo and $2 S$ positions in reconstructed phases [Figs. S6(a)-S6(e)] using both experimental and simulated hollow diffraction patterns, for various inner angles. The lines shown in Figs. S7(a) and S7(b) show results with 3-pixel widths extracted across $\mathrm{Mo}$ and $2 S$ positions indicated by red arrows of top-right and bottom-right insets to Figs. S6(a)S6(e), respectively. The line profiles extracted from both experimental and simulated data show similar trends. In general, as the hollow inner angle increased, the value of reconstructed phases decreases together with the overall quality of reconstructions. The absolute phase values in the simulations were larger (by 3\%) than those in the experiments, which may be due to a small Stobbs factor present for data recorded from a monolayer sample [40]. It should be noted that the reconstructions using hollow angles of less than $10 \mathrm{mrad}$ showed similar phase values to those obtained from conventional ptychography. For hollow angles larger than $10 \mathrm{mrad}$, the phase values decreased abruptly, and the (300) reflection was not present [Figs. S6(f)-S6(j)]. In addition, given the oversampling ratio used [41], the degree of the redundancy in our experimental ptychographic data sets for different hollow angles is shown in Fig. S8. It is clear that for the increasing hollow inner angle, the degree of redundancy is decreased. Overall, these results indicate that an inner angle of less than $10 \mathrm{mrad}$ is necessary to reconstruct resolved data.

Finally, we have compared experimental and simulated diffraction patterns after 50 reconstructed iterations, as shown in Figs. 2(f)-2(j) and 2(k)-2(o), respectively, demonstrating that after 50 iterations, the information lost in the hollow area was retrieved. The accuracy of the missing information recovered as a function of the number of iterations was quantified using the root mean square error 
(RMSE) calculated from the hollow area, as shown in Fig. S9. As expected, this shows that larger inner angles give slower convergence in the reconstructions. However, notably for inner angles up to $15 \mathrm{mrad}$, the RMSE did not deviate substantially after 50 iterations.

Effects of other parameters.-For typical atomic resolution EELS mapping, the pixel dwell time for a spectrum is generally much longer (of the order of milliseconds $[3,4]$ ) than that for the ADF imaging (of the order of microseconds [42,43]). Considering the total acquisition time for EELS spectrum imaging, the sampling or scan step size used for EELS mapping $(0.5 \AA$ or larger as reported in $[3,44])$ is generally much coarser than that used for high resolution ADF imaging $(0.07 \AA$ as reported in $[43,45])$. Hence, HAADF images acquired simultaneously with a spectrum image are undersampled, thereby setting an additional experimental resolution limit. In addition, as a recent developed STEM technique, DPC with annularsegmented detectors can potentially be combined with EELS. However, in a similar undersampled condition, the resolution of the simultaneously acquired DPC image is limited by the sampling or scan step size [Figs. S10(k)$\mathrm{S} 10(\mathrm{o})]$. More detailed discussions are provided in the Supplemental Material, part V [31]. It is clear that DPC images with a step size of $0.612 \AA$ [Figs. S10(k)-S10(o)] are completely pixelated and the high resolution information is lost.

In the configuration described here, the coupling between EELS and the hollow ptychographic reconstruction allows atomic resolution in the reconstructed phase with simultaneous atomic resolution EELS mapping. This is possible because the resolution in reconstructed phases is independent of the scan step size and is instead determined by the maximum collection angle of the detector used to record diffraction patterns $[19,29]$.

In the experiments described here, the probe overlap ratio between adjacent positions was selected to give an equivalent step size to that used in a typical atomic resolution EELS mapping experiment. It is, therefore, useful to investigate the effect of scan step size on both the convergence and resolution of the ptychographic reconstruction [46,47]. The position accuracy of the scanned probe can also significantly affect the reconstructions [47], and to account for this, we also explored the effects of few-pixel position errors on the reconstructions.

As already described, the experimental data consisted of a $256 \times 256$ array of diffraction patterns with a step size of $0.204 \AA$. To explore the effect of step size on the hollow ptychographic reconstruction, only every second or third diffraction pattern was selected to synthesize data sets with an effective scan step of 0.408 or $0.612 \AA$, corresponding to overlap ratios of $79 \%$ or $58 \%$. Furthermore, a 2-pixel random position error was introduced at each scan position used in the reconstruction. The reconstructed phases with different steps and with or without the introduction of a

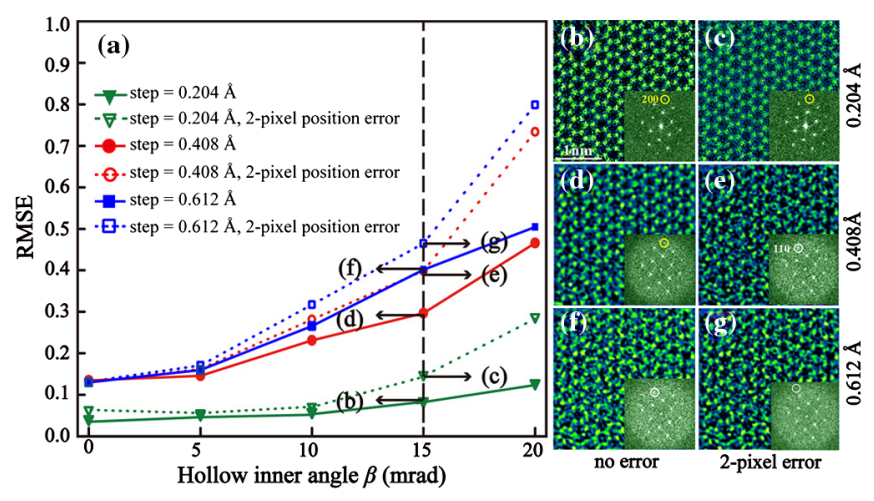

FIG. 4. (a) Root mean square error (RMSE) as a function of hollow inner angle $\beta$ for various step sizes and position errors. Solid lines represent step sizes of $0.204 \AA$ (-filled inverted triangle-), $0.408 \AA$ (-filled circle-), and $0.612 \AA$ (-filled rectangle-) without the introduction of a probe position error. Same color dotted lines represent the results with 2-pixel random error introduced at the probe positions. (b)-(g) Reconstructed phases for the conditions marked by the dashed vertical line in (a). The $1-\mathrm{nm}$ scale bar applies to all images in (b)-(g). The associated power spectra are insets to (b)-(g), respectively. The yellow and white circles mark (200) and (110) reflections corresponding to spacings of 136 and $158 \mathrm{pm}$, respectively.

probe position error are shown in Fig. S11. The quality of reconstructions was evaluated using the RMSE calculated from the entire illuminated area after 50 iterations. Figure 4(a) shows the RMSE as a function of hollow inner angle for various conditions. The RMSE of reconstructions clearly increases with increasing hollow inner angle, step size, or position error visible in the degraded reconstruction phase quality [Figs. 4(b)-4(g)]. For a hollow inner angle less than $15 \mathrm{mrad}$, the change in RMSE with or without probe position error was negligible. However, when the hollow angle was increased to $15 \mathrm{mrad}$, the tolerance to position error was reduced as a smaller portion of the signal in diffraction patterns was used in the reconstructions. However, the position error can be corrected using a suitable high resolution scan position refinement [19] to enable the recovery of high resolution structural information at high resolution using a large hollow angle. As observed in Figs. 4(b)-4(g), for hollow angles up to $15 \mathrm{mrad}$, the reconstruction is relatively insensitive to the scan step and even with a step of $0.612 \AA$ ( $58 \%$ overlap ratio), a resolution of $1.58 \AA$ was still achieved in the reconstruction, as indicated by the presence of the (110) reflection in the power spectra.

For a better understanding of the performance of hollow ptychography with respect to the specimen thickness, we have carried out simulations of a series of specimen thicknesses up to $215 \AA$ for a multilayer $\mathrm{MoS}_{2}$ crystal, as shown in Fig. S12. It can be seen that regardless of the size of the hollow angle the phase of the atomic columns starts to wrap and contrast reversal occurs as the specimen thickness increases beyond $71 \AA$, and the phase further wraps as thickness increases. This thickness-dependent 
contrast is consistent with that observed in conventional ptychography [48].

The ptychographic results reported here show that with a 24-mrad probe convergence semiangle, electrons scattered within a semiangle up to $15 \mathrm{mrad}$ can removed from ptychographic reconstructions without degrading the resolution significantly. These low-angle scattered electrons could be allowed to enter an EELS spectrometer to perform simultaneous chemical analysis [49]. Given that inelastic scattering is concentrated at smaller angles [49], particularly for low atomic number elements (and correspondingly low-energy inner shell levels), a spectrometer with a limited acceptance angle can still collect an appreciable fraction of transmitted loss electrons [50]. This geometry therefore allows ptychographic reconstruction of weakly scattering samples containing low-atomic-number elements (such as biological materials) with high contrast at atomic resolution correlated with simultaneous chemical analysis. Such highresolution correlation information cannot easily be directly obtained using alternative experimental approaches, e.g., two-step schemes involving fast detectors $[18,29]$ in conjunction with energy filters [49].

Generally, in comparison to existing phase contrast imaging methods in STEM (such as DPC), current reconstruction algorithms and limitations in computational speed do not allow real time reconstruction. However, ptychography can retrieve quantifiable phase information at high resolution [25,51], even beyond the Abbe limit [29] and the hollow geometry allows it to couple simultaneously with EELS chemical mapping, even under undersampled conditions.

Conclusions. - We have demonstrated that a hollow ptychographic imaging configuration can enable simultaneous coupling of ptychography and EELS mapping. We have also shown that hollow diffraction patterns can be used to reconstruct the phase of monolayer $\mathrm{MoS}_{2}$ at sub- $\AA$ resolution. When compared to conventional ADF imaging at an equivalent dose, hollow ptychographic reconstruction provides improved resolution, lower noise, and higher contrast for light atoms. Although the hollow configuration uses a lower signal in the diffraction patterns than conventional ptychography, it remains robust to an oversampling of 58\% and 2-pixel probe position error up to a $15-\mathrm{mrad}$ inner angle for a resolution of $1.58 \AA$. In the future, with the development of fast hollow detectors that are compatible with an EELS spectrometer, we anticipate that this geometry will enable simultaneous, correlative analysis using both highly sensitive phase information and chemical mapping.

P.W., B.S., and Z.D. acknowledge funding from the National Natural Science Foundation of China (No. 11474147 No. 11874199), the National Basic Research Program of China (Grant No. 2015CB654901), the Natural Science Foundation of Jiangsu Province (Grant No. BK20151383), and the Fundamental Research Funds for the Central Universities (No. 020514380136). F.Z. acknowledges funding from the National Natural Science Foundation of China (No. 11775105). We thank Diamond Light Source for access and support in the use of the electron Physical Science Imaging Centre (Instrument E02, EM17918) that contributed to the results presented. A. I. K. acknowledges financial support from EPSRC (Platform Grant No. EP/K032518/1).

* Corresponding author. wangpeng@nju.edu.cn

[1] S. J. Pennycook and P. D. Nellist, Scanning Transmission Electron Microscopy (Springer, New York, 2011).

[2] K. Kimoto, T. Asaka, T. Nagai, M. Saito, Y. Matsui, and K. Ishizuka, Nature (London) 450, 702 (2007).

[3] D. A. Muller, L. F. Kourkoutis, M. Murfitt, J. H. Song, H. Y. Hwang, J. Silcox, N. Dellby, and O. L. Krivanek, Science 319, 1073 (2008).

[4] D. A. Muller, Nat. Mater. 8, 263 (2009).

[5] P. D. Nellist, M. F. Chisholm, N. Dellby, O. L. Krivanek, M. F. Murfitt, Z. S. Szilagyi, A. R. Lupini, A. Borisevich, and W. H. Sides Jr., and S. J. Pennycook, Science 305, 1741 (2004).

[6] N. Shibata, S. D. Findlay, Y. Kohno, H. Sawada, Y. Kondo, and Y. Ikuhara, Nat. Phys. 8, 611 (2012).

[7] W. Hoppe, Acta Crystallogr. Sect. A 25, 495 (1969).

[8] O. Bunk, M. Dierolf, S. Kynde, I. Johnson, O. Marti, and F. Pfeiffer, Ultramicroscopy 108, 481 (2008).

[9] J. M. Rodenburg, Advances in Imaging and Electron Physics (Elsevier, New York, 2008).

[10] A. M. Maiden and J. M. Rodenburg, Ultramicroscopy 109, 1256 (2009).

[11] T. M. Godden, R. Suman, M. J. Humphry, J. M. Rodenburg, and A. M. Maiden, Opt. Express 22, 12513 (2014).

[12] P. Thibault, M. Dierolf, A. Menzel, O. Bunk, C. David, and F. Pfeiffer, Science 321, 379 (2008).

[13] A. M. Maiden, G. R. Morrison, B. Kaulich, A. Gianoncelli, and J. M. Rodenburg, Nat. Commun. 4, 1669 (2013).

[14] F. Zhang, I. Peterson, J. Vila-Comamala, A. Diaz, F. Berenguer, R. Bean, B. Chen, A. Menzel, I. K. Robinson, and J. M. Rodenburg, Opt. Express 21, 13592 (2013).

[15] M. J. Humphry, B. Kraus, A. C. Hurst, A. M. Maiden, and J. M. Rodenburg, Nat. Commun. 3, 730 (2012).

[16] C. T. Putkunz et al., Phys. Rev. Lett. 108, 073901 (2012).

[17] A. J. D'Alfonso, A. J. Morgan, A. W. C. Yan, P. Wang, H. Sawada, A. I. Kirkland, and L. J. Allen, Phys. Rev. B 89, 064101 (2014).

[18] H. G. Brown, A. J. D’Alfonso, Z. Chen, A. J. Morgan, M. Weyland, C. Zheng, M. S. Fuhrer, S. D. Findlay, and L. J. Allen, Phys. Rev. B 93, 134116 (2016).

[19] P. Wang, F. Zhang, S. Gao, M. Zhang, and A. I. Kirkland, Sci. Rep. 7, 2857 (2017).

[20] S. Gao, P. Wang, F. Zhang, G. T. Martinez, P. D. Nellist, X. Pan, and A. I. Kirkland, Nat. Commun. 8, 163 (2017).

[21] J. M. Rodenburg and R. H. T. Bates, Phil. Trans. R. Soc. A 339, 521 (1992).

[22] P. D. Nellist, B. C. McCallum, and J. M. Rodenburg, Nature (London) 374, 630 (1995). 
[23] T. J. Pennycook, A. R. Lupini, H. Yang, M. F. Murfitt, L. Jones, and P. D. Nellist, Ultramicroscopy 151, 160 (2015).

[24] H. Yang, T. J. Pennycook, and P. D. Nellist, Ultramicroscopy 151, 232 (2015).

[25] A. M. Maiden, M. C. Sarahan, M. D. Stagg, S. M. Schramm, and M. J. Humphry, Sci. Rep. 5, 14690 (2015).

[26] H. Yang et al., Nat. Commun. 7, 12532 (2016).

[27] H. Yang, P. Ercius, P. D. Nellist, and C. Ophus, Ultramicroscopy 171, 117 (2016).

[28] C. Ophus, J. Ciston, J. Pierce, T. R. Harvey, J. Chess, B. J. McMorran, C. Czarnik, H. H. Rose, and P. Ercius, Nat. Commun. 7, 10719 (2016).

[29] Y. Jiang et al., Nature (London) 559, 343 (2018).

[30] J. A. Mir et al., Ultramicroscopy 182, 44 (2017).

[31] See Supplemental Material at http://link.aps.org/ supplemental/10.1103/PhysRevLett.121.146101 for additional experimental and simulation methods information, large data sets of images used to obtain results, and supporting calculations of data redundancy and simulations, which includes Refs. [19,25,29,32-38].

[32] S. Cao, P. Kok, P. Li, A. M. Maiden, and J. M. Rodenburg, Phys. Rev. A 94, 063621 (2016).

[33] P. Thibault and A. Menzel, Nature (London) 494, 68 (2013).

[34] P. Li, T. Edo, D. Batey, J. Rodenburg, and A. Maiden, Opt. Express 24, 9038 (2016).

[35] V. K. Loane, P. Xu, and J. Solcox, Acta Crystallogr. Sect. A 47, 267 (1991).

[36] V. K. Pecharsky and P. Y. Zavalij, Fundamentals of Powder Diffraction and Structural Characterization of Materials (Springer, New York, 2009).
[37] X. Huang, H. Miao, J. Steinbrener, J. Nelson, D. Shapiro, A. Stewart, J. Turner, and C. Jacobsen, Opt. Express 17, 13541 (2009).

[38] J. E. Kirkland, Advanced Computing in Electron Microscopy (Springer, New York, 2010).

[39] F. Hue, J. M. Rodenburg, A. M. Maiden, and P. A. Midgley, Ultramicroscopy 111, 1117 (2011).

[40] M. J. Hytch and W. M. Stobbs, Ultramicroscopy 53, 191 (1994).

[41] A. M. Maiden, J. H. Marti, F. Zhang, and M. R. John, J. Opt. Soc. Am. 28, 604 (2011).

[42] R. Erni, M. D. Rossell, C. Kisielowski, and U. Dahmen, Phys. Rev. Lett. 102, 096101 (2009).

[43] H. Sawada, Y. Tanishiro, N. Ohashi, T. Tomita, F. Hosokawa, T. Kaneyama, Y. Kondo, and K. Takayanagi, J. Electron Microsc. 58, 357 (2009).

[44] H. L. Xin, C. Dwyer, and D. A. Muller, Ultramicroscopy 139, 38 (2014).

[45] T. Akita, K. Tanaka, and M. Kohyama, J. Mater. Sci. 43, 3917 (2008).

[46] X. Huang, H. Yan, R. Harder, Y. Hwu, I. K. Robinson, and Y. S. Chu, Opt. Express 22, 12634 (2014).

[47] A. Tripathi, I. McNulty, and O. G. Shpyrko, Opt. Express 22, 1452 (2014).

[48] H. Yang et al., Ultramicroscopy 180, 173 (2017).

[49] R. F. Egerton, Electron Energy Loss Spectroscopy (Springer, New York, 2011).

[50] M. Isaacson and D. Johnson, Ultramicroscopy 1, 33 (1975).

[51] F. Hüe, J. M. Rodenburg, A. M. Maiden, F. Sweeney, and P. A. Midgley, Phys. Rev. B 82, 121415 (2010). 